\title{
Pacific
}

Journal of

Mathematics

\section{NONPLANARITY OF UNIT GRAPHS AND CLASSIFICATION OF THE TOROIDAL ONES}

A. K. Das, H. R. Maimani, M. R. Pournaki and S. Yassemi 


\title{
NONPLANARITY OF UNIT GRAPHS AND CLASSIFICATION OF THE TOROIDAL ONES
}

\author{
A. K. Das, H. R. Maimani, M. R. Pournaki and S. Yassemi
}

\begin{abstract}
The unit graph of a ring $R$ with nonzero identity is the graph in which the vertex set is $\boldsymbol{R}$, and two distinct vertices $\boldsymbol{x}$ and $\boldsymbol{y}$ are adjacent if and only if $x+y$ is a unit in $R$. In this paper, we derive several necessary conditions for the nonplanarity of the unit graphs of finite commutative rings with nonzero identity, and determine, up to isomorphism, all finite commutative rings with nonzero identity whose unit graphs are toroidal.
\end{abstract}

\section{Introduction}

Algebraic combinatorics is an area of mathematics which employs methods of abstract algebra in various combinatorial contexts and vice versa. Associating a graph to an algebraic structure is a research subject in this area and has attracted considerable attention. The research in this subject aims at exposing the relationship between algebra and graph theory and at advancing the application of one to the other. In fact, there are three major problems in this area: (1) characterization of the resulting graphs, (2) characterization of the algebraic structures with isomorphic graphs, and (3) realization of the connections between the algebraic structures and the corresponding graphs. Beck [1988] introduced the idea of a zero-divisor graph of a commutative ring $R$ with nonzero identity. He defined $\Gamma_{0}(R)$ to be the graph in which the vertex set is $R$, and two distinct vertices $x$ and $y$ are adjacent if and only if $x y=0$. He was mostly concerned with coloring of $\Gamma_{0}(R)$. Beck conjectured that $\chi(R)=\omega(R)$, where $\chi(R)$ and $\omega(R)$ denote, respectively, the chromatic number and the clique number of $\Gamma_{0}(R)$. Such graphs are called weakly perfect graphs. This investigation of coloring of a commutative ring was then continued by Anderson and Naseer [1993]. They gave a counterexample for the above conjecture of Beck. Anderson and Livingston [1999] proposed a different

This research was in part supported by a grant from IPM (H. R. Maimani: No. 91050214, M. R. Pournaki: No. 92130115, and S. Yassemi: No. 92130214), by a grant from INSF (M. R. Pournaki), and by a grant from the Center for International Scientific Studies and Collaboration (CISSC), Iran, and Égide - Programme Gundishapur 2012, Hubert Curien Partnership, France (M. R. Pournaki and S. Yassemi: No. 27462PL).

MSC2010: primary 05C75; secondary 13M05.

Keywords: finite commutative ring, unit graph, genus. 
method of associating a zero-divisor graph to a commutative ring $R$, and according to them this gives a better illustration of the zero-divisor structure of the ring. They defined $\Gamma(R)$ to be the graph in which the vertex set consists of all the nonzero zero-divisors of $R$, and two distinct vertices $x$ and $y$ are adjacent if and only if $x y=0$. For a survey and recent results concerning zero-divisor graphs, we refer the reader to [Anderson et al. 2011]. In literature, one can find a number of different types of graphs attached to rings or other algebraic structures. For a survey of recent results concerning graphs attached to rings, we refer the reader to [Maimani et al. 2011a].

The present paper deals with what is known as the unit graph of a ring, a notion that generalizes the idea of Grimaldi [1990] who introduced and studied in detail a graph $G\left(\mathbb{Z}_{n}\right)$ in which the vertex set is the ring $\mathbb{Z}_{n}$ of integers modulo a positive integer $n$, and two distinct vertices $x$ and $y$ are adjacent if and only if $x+y$ is a unit in $\mathbb{Z}_{n}$. In general, given an arbitrary ring $R$ with nonzero identity, its unit graph $G(R)$ is defined to be the graph in which the vertex set is $R$, and two distinct vertices $x$ and $y$ are adjacent if and only if $x+y$ is a unit in $R$. Some of the properties of this graph have been studied in detail in [Ashrafi et al. 2010; Maimani et al. 2010a; 2010b; 2010c; 2011b]. The graphs in Figure 1 are the unit graphs of the rings indicated.
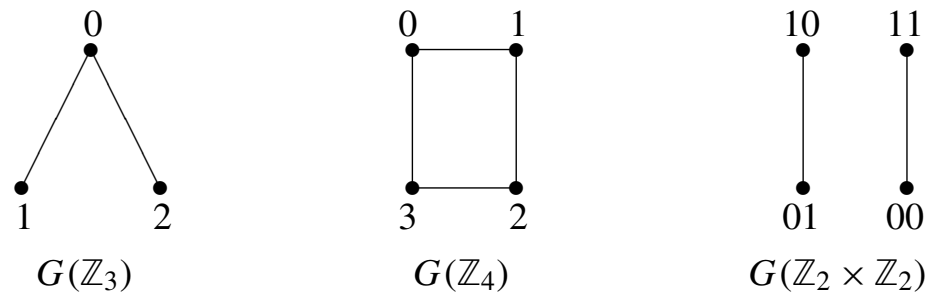

Figure 1. The unit graphs of some specific rings.

It is easy to see that, given any two rings $R$ and $S$, if $R \cong S$ as rings, then $G(R) \cong G(S)$ as graphs. This point is illustrated in Figure 2 for the unit graphs of two isomorphic rings $\mathbb{Z}_{3} \times \mathbb{Z}_{2}$ and $\mathbb{Z}_{6}$.

It is also easy to see that if the rings $R_{1}, R_{2}, S_{1}$ and $S_{2}$ are such that $G\left(R_{1}\right) \cong$ $G\left(R_{2}\right)$ and $G\left(S_{1}\right) \cong G\left(S_{2}\right)$, then $G\left(R_{1} \times S_{1}\right) \cong G\left(R_{2} \times S_{2}\right)$. However, this property does not hold in general for other widely studied graphs associated to rings (for example, the zero-divisor graphs).

In this paper, we derive several necessary conditions for the nonplanarity of the unit graphs of finite commutative rings with nonzero identity; in particular, we show that given any positive integer $g$, there exists only a finite number of finite commutative rings with nonzero identity whose unit graphs have genus $g$. 


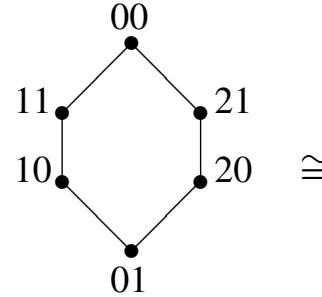

$G\left(\mathbb{Z}_{3} \times \mathbb{Z}_{2}\right)$

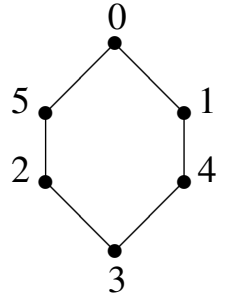

$G\left(\mathbb{Z}_{6}\right)$

Figure 2. The unit graphs of two isomorphic rings.

Also, in analogy with the results in [Maimani et al. 2012], we determine, up to isomorphism, all finite commutative rings with nonzero identity whose unit graphs are toroidal. It may be recalled here that the genus of a graph $G$, denoted by $\gamma(G)$, is smallest nonnegative integer $g$ such that the graph $G$ can be embedded on the surface obtained by attaching $g$ handles to a sphere. The graphs of genus 0 and 1 are called planar graphs and toroidal graphs respectively. For unexplained terminology and notations in this paper, we refer the reader to [Chartrand and Oellermann 1993].

\section{Some auxiliary results and the related concepts}

In this section, we put together certain graph theoretical terminologies and some well-known results which have been used extensively in the forthcoming sections. Note that all graphs considered in this section are finite simple graphs, that is, graphs with finitely many vertices and without loops or multiple edges.

Let $x$ and $y$ be any two vertices in a graph $G$. Then, $x$ and $y$ are said to be adjacent in $G$ if $x \neq y$ and there is an edge $\{x, y\}$ between $x$ and $y$. A path between $x$ and $y$ is a sequence $\left\{x, x_{1}\right\},\left\{x_{1}, x_{2}\right\}, \ldots,\left\{x_{n}, y\right\}$ of distinct edges, which is also written as $\left\{x, x_{1}, x_{2}, \ldots, x_{n}, y\right\}$, where the vertices $x, x_{1}, x_{2}, \ldots, x_{n}, y$ are all distinct (except, possibly, $x$ and $y$ ). A path between $x$ and $y$ is called a cycle if $x=y$. The number of edges in a path or a cycle, is called its length.

A graph $G$ is said to be connected if there is a path between every pair of distinct vertices in $G$. A chord of a cycle in a graph is an edge of the graph which does not lie in the edge set of the cycle but whose endpoints lie in the vertex set of the cycle. A chordless cycle of a graph is a cycle without any chord.

A cycle of a graph, embedded on a surface, is called contractible with respect to the embedding if it can be contracted continuously on the surface to a point. A cycle of a toroidal graph is said to be flat if it is contractible in every torus embedding of the graph. Given a cycle $C$ of a graph $G$, we write $G-C$ to denote the graph obtained from $G$ by deleting the vertices of $C$ and the edges of the graph incident to the vertices of $C$. 
A graph $G$ is said to be complete if there is an edge between every pair of distinct vertices in $G$. We denote the complete graph with $n$ vertices by $K_{n}$. A bipartite graph is the one whose vertex set can be partitioned into two disjoint parts in such a way that the two end vertices of every edge lie in different parts. Among the bipartite graphs, the complete bipartite graph is the one in which two vertices are adjacent if and only if they lie in different parts. The complete bipartite graph, with parts of size $m$ and $n$, is denoted by $K_{m, n}$.

A subdivision of an edge $\{x, y\}$ in a graph is a path $\left\{x, x_{1}, x_{2}, \ldots, x_{n}, y\right\}$ obtained by inserting some new vertices $x_{1}, x_{2}, \ldots, x_{n}$ into the edge $\{x, y\}$. A subdivision of a graph $G$ is the result of some subdivisions of the edges of $G$. Furthermore, every graph can be considered as a subdivision of itself. A remarkably simple characterization of planar graphs was given by Kuratowski in 1930. Kuratowski's theorem [Chartrand and Oellermann 1993, page 153] says that a graph is planar if and only if it contains no subdivision of $K_{3,3}$ or $K_{5}$. As a consequence of Kuratowski's theorem, one has the following result.

Lemma 2.1 [Neufeld and Myrvold 1997, Theorem 2.1]. If a cycle $C$ of a toroidal graph $G$ is such that $G-C$ is nonplanar, then $C$ is flat in $G$. Furthermore, if flat $C$ is chordless and $G-C$ is connected, then $C$ is a flat face in any torus embedding of $G$.

Given a graph $G$, we denote its vertex set by $V(G)$ and its edge set by $E(G)$. If $G_{1}$ and $G_{2}$ are any two graphs, then their disjoint union, denoted by $G_{1} \sqcup G_{2}$, is defined to be the graph in which the vertex set is $V\left(G_{1}\right) \sqcup V\left(G_{2}\right)$ and the edge set is $E\left(G_{1}\right) \sqcup E\left(G_{2}\right)$. The following result, which follows from [Battle et al. 1962, Corollary 2], often enables us to reformulate some results which are otherwise true for connected graphs.

Lemma 2.2. If a graph $G$ is isomorphic to the disjoint union $G_{1} \sqcup G_{2}$ of two graphs $G_{1}$ and $G_{2}$, then $\gamma(G)=\gamma\left(G_{1}\right)+\gamma\left(G_{2}\right)$.

If $G$ is a graph and $x \in V(G)$, then the degree of $x$ in $G$ is defined as the number of vertices adjacent to $x$ in $G$, and is denoted by $\operatorname{deg}(x)$. If $r$ is a nonnegative integer such that $\operatorname{deg}(x)=r$ for all $x \in V(G)$, then the graph $G$ is said to be $r$-regular. In general, we write $\delta(G)$ to denote the minimum of the degrees of the vertices of $G$. In this connection, using Lemma 2.2, one may reformulate [Wickham 2008, Proposition 2.1] as follows.

Lemma 2.3. If $G$ is a graph (not necessarily connected) having $n$ vertices with $n \geq 3$, then

$$
\delta(G) \leq 6+\frac{12(\gamma(G)-1)}{n} .
$$

The girth of a graph $G$ is the minimum of the lengths of all cycles in $G$, and is denoted by $\operatorname{gr}(G)$. If $G$ is acyclic, that is, if $G$ has no cycles, then we write 
$\operatorname{gr}(G)=\infty$. It has been proved in [Archdeacon 1996, Section 2.3] that if $G$ is a connected graph (but not acyclic) having $n$ vertices and $m$ edges, then

$$
\gamma(G) \geq \frac{m(k-2)}{2 k}-\frac{n}{2}+1,
$$

where $k=\operatorname{gr}(G)$. Therefore, using the facts that in an acyclic graph the total number of edges is less than the total number of vertices, and that the girth of a bipartite graph (which is not acyclic) is at least four, we have, in view of Lemma 2.2, the following result.

Lemma 2.4. If $G$ is a bipartite graph (not necessarily connected) having $n$ vertices and $m$ edges with $n \geq 3$, then

$$
\gamma(G) \geq \frac{m}{4}-\frac{n}{2}+1
$$

We conclude the section with two useful results.

Lemma 2.5 [White 1973, Theorem 6-38]. If $n \geq 3$, then

$$
\gamma\left(K_{n}\right)=\left\lceil\frac{(n-3)(n-4)}{12}\right\rceil .
$$

Lemma 2.6 [White 1973, Theorem 6-37]. If $m, n \geq 2$, then

$$
\gamma\left(K_{m, n}\right)=\left\lceil\frac{(m-2)(n-2)}{4}\right\rceil .
$$

\section{Some necessary conditions for the nonplanarity of unit graphs}

In this section, we derive a few necessary conditions for the nonplanarity of the unit graphs of finite commutative rings with nonzero identity. However, we begin with a known result.

Lemma 3.1 [Ashrafi et al. 2010, Proposition 2.4]. Let $R$ be a finite commutative ring with nonzero identity, and $U(R)$ be the set of all unit elements of $R$. Let $x \in R$. Then

$$
\operatorname{deg}(x)= \begin{cases}|U(R)|-1 & \text { if } x \in U(R) \text { and } 2 \in U(R), \\ |U(R)| & \text { otherwise. }\end{cases}
$$

Let us now derive the first necessary condition for the nonplanarity of unit graphs.

Proposition 3.2. Let $R$ be a finite commutative ring with nonzero identity such that $\gamma(G(R))=g>0$. Then either $|R| \leq 12(g-1)$ or $|U(R)| \leq 7$. 


\begin{tabular}{|cccc|}
\hline$|U(R)||Z(R)|$ & $|R|$ & $R$ \\
\hline 7 & 1 & 8 & $\mathbb{F}_{8}$ \\
6 & 1 & 7 & $\mathbb{Z}_{7}$ \\
6 & 3 & 9 & $\mathbb{Z}_{9}, \mathbb{Z}_{3}[x] /\left\langle x^{2}\right\rangle$ \\
4 & 1 & 5 & $\mathbb{Z}_{5}$ \\
4 & 4 & 8 & $\mathbb{Z}_{8}, \mathbb{Z}_{2}[x] /\left\langle x^{3}\right\rangle, \mathbb{Z}_{2}[x] /\left\langle 2 x, x^{2}-2\right\rangle$, \\
3 & 1 & 4 & $\mathbb{Z}_{2}[x, y] /\langle x, y\rangle^{2}, \mathbb{Z}_{4}[x] /\langle 2, x\rangle^{2}$ \\
2 & 1 & 3 & $\mathbb{F}_{4}$ \\
2 & 2 & 4 & $\mathbb{Z}_{3}$ \\
1 & 1 & 2 & $\mathbb{Z}_{4}, \mathbb{Z}_{2}[x] /\left\langle x^{2}\right\rangle$ \\
\hline
\end{tabular}

Table 1. Finite commutative local rings with at most 7 units.

Proof. If $|R|>12(g-1)$, then, using Lemma 2.3, we deduce that

$$
\delta(G(R)) \leq 6+\frac{12(g-1)}{|R|}<6+1=7 .
$$

Since $\delta(G(R))$ is an integer, we have $\delta(G(R)) \leq 6$. Hence, it follows from Lemma 3.1 that $|U(R)| \leq 7$.

Let $R$ be a finite commutative ring with nonzero identity. Let $Z(R)$ denote the set of all zero-divisors of $R$. It is easy to see that $U(R) \sqcup Z(R)=R$ and so $|U(R)|+|Z(R)|=|R|$. The structure theorem for finite commutative rings says that $R$ is isomorphic to a direct product of finite commutative local rings with nonzero identity, and such a product is unique up to the order in which the factors are arranged (see [McDonald 1974]). If $R$ itself is a local ring, then we have the following result which is essentially due to Raghavendran.

Lemma 3.3 [Raghavendran 1969, Theorem 2]. Let $R$ be a finite commutative local ring with nonzero identity. Then $|R|=p^{n r},|Z(R)|=p^{(n-1) r}$ and $|U(R)|=$ $p^{(n-1) r}\left(p^{r}-1\right)$ for some prime $p$ and some positive integers $n$ and $r$.

Now, Lemma 3.3 together with some well-known results on the structures of small local rings (see, for example, [Corbas and Williams 2000a; 2000b]) enable us to obtain the following result.

Proposition 3.4. Let $R$ be a finite commutative local ring with nonzero identity such that $|U(R)| \leq 7$. Then the possible forms of $R$ are given by Table 1 .

As a consequence of the above result, we derive a necessary condition for the nonplanarity of the unit graphs of finite commutative local rings with nonzero identity. 
Corollary 3.5. Let $R$ be a finite commutative local ring with nonzero identity such that $\gamma(G(R))=g>0$. Then $|R| \leq \max \{9,12(g-1)\}$. In particular, the number of finite commutative local rings with nonzero identity such that $\gamma(G(R))=g>0$ is finite.

Proof. If $|R| \leq 12(g-1)$, then we are done. Otherwise, by Proposition 3.2, we have $|U(R)| \leq 7$. Therefore, by Proposition 3.4, $|R| \leq 9$. This completes the proof of the first part. The last part of the corollary is obvious, because, given any positive integer $g$, the number of rings $R$ with $|R| \leq \max \{9,12(g-1)\}$ is clearly finite.

In Figure 1, one can see that $G\left(\mathbb{Z}_{2} \times \mathbb{Z}_{2}\right)$ is isomorphic to the disjoint union of two copies of $G\left(\mathbb{Z}_{2}\right)$. In fact, it is not difficult to make a more general observation that if $S$ is a finite commutative ring with nonzero identity, then the unit graph $G\left(\mathbb{Z}_{2} \times \mathbb{Z}_{2} \times S\right)$ is isomorphic to the disjoint union of two copies of the unit graph $G\left(\mathbb{Z}_{2} \times S\right)$. Therefore, in view of Lemma 2.2, we have the following result.

Lemma 3.6. Let $S$ be a finite commutative ring with nonzero identity. Then we have $\gamma\left(G\left(\mathbb{Z}_{2} \times \mathbb{Z}_{2} \times S\right)\right)=2 \gamma\left(G\left(\mathbb{Z}_{2} \times S\right)\right)$. In particular, $\gamma\left(G\left(\left(\mathbb{Z}_{2}\right)^{t}\right)\right)=0$ for all $t \geq 1$.

The following result plays an important role in getting rid of all finite commutative rings with nonzero identity whose unit graphs are planar.

Lemma 3.7 [Ashrafi et al. 2010, Theorem 5.14]. Let $R$ be a finite commutative ring with nonzero identity. Then the unit graph $G(R)$ is planar if and only if $R$ is isomorphic to one of $\mathbb{Z}_{5}, \mathbb{Z}_{3} \times \mathbb{Z}_{3}$ or $\left(\mathbb{Z}_{2}\right)^{t} \times S$, where $t \geq 0$ and $S$ is one of the rings $\mathbb{Z}_{2}, \mathbb{Z}_{3}, \mathbb{Z}_{4}, \mathbb{F}_{4}$ and $\left\{\left[\begin{array}{cc}a & b \\ 0 & a\end{array}\right] \mid a, b \in \mathbb{Z}_{2}\right\} \cong \mathbb{Z}_{2}[x] /\left\langle x^{2}\right\rangle$.

We are now in a position to state and prove the main result of this section.

Theorem 3.8. Let $R$ be a finite commutative ring with nonzero identity such that $\gamma(G(R))=g>0$. Then either

$$
|R| \leq 12(g-1) \quad \text { or } \quad R \cong\left(\mathbb{Z}_{2}\right)^{t} \times S,
$$

where $0 \leq t \leq 1+\log _{2} g$ and $S$ is one of the finite rings given by Table 2 .

Proof. Let $|R|>12(g-1)$. In this case, Proposition 3.2 implies that $|U(R)| \leq$ 7. Using the structure theorem for finite commutative rings (see the discussion preceding Lemma 3.3) along with Lemma 3.6 and the fact that $g>0$, we conclude that

$$
R \cong\left(\mathbb{Z}_{2}\right)^{t} \times R_{1} \times R_{2} \times \cdots \times R_{k},
$$

where $0 \leq t \leq 1+\log _{2} g, k \geq 1$ and each $R_{i}$ is a finite commutative local ring with nonzero identity having at least three elements. Now, we have

$$
|U(R)|=\left|U\left(R_{1} \times R_{2} \times \cdots \times R_{k}\right)\right|=\left|U\left(R_{1}\right)\right| \times\left|U\left(R_{2}\right)\right| \times \cdots \times\left|U\left(R_{k}\right)\right| .
$$


Clearly, $|U(R)| \neq 1$. Since $|U(R)| \leq 7$, we have the following possibilities:

(1) $|U(R)|=p$, where $p=2,3,5$ or 7. In this case, we have $k=1$ and $\left|U\left(R_{1}\right)\right|=p$.

(2) $|U(R)|=6$. In this case, either we have $k=1$ and $\left|U\left(R_{1}\right)\right|=6$, or we have $k=2,\left|U\left(R_{1}\right)\right|=2$ and $\left|U\left(R_{2}\right)\right|=3$.

(3) $|U(R)|=4$. In this case, either we have $k=1$ and $\left|U\left(R_{1}\right)\right|=4$, or we have $k=2,\left|U\left(R_{1}\right)\right|=2$ and $\left|U\left(R_{2}\right)\right|=2$.

The result now follows from Proposition 3.4 and Lemma 3.7.

As an immediate corollary, we have the following result.

Corollary 3.9. Let $R$ be a finite commutative ring with nonzero identity such that $\gamma(G(R))=g>0$. Then $|R| \leq 32 g$. In particular, given any positive integer $g$, the number of finite commutative rings with nonzero identity such that $\gamma(G(R))=g$ is finite.

Proof. By Theorem 3.8, either $|R| \leq 12(g-1)$ or $R \cong\left(\mathbb{Z}_{2}\right)^{t} \times S$, where $0 \leq$ $t \leq 1+\log _{2} g$ and $S$ is a ring with $|S| \leq 16$. In the second case, $|R|=2^{t}|S|=$ $2^{t-1}(2|S|) \leq 32 g$. Hence, it follows that $|R| \leq \max \{32 g, 12(g-1)\}=32 g$. The last part of the corollary is obvious, because, given any positive integer $g$, the number of rings $R$ with $|R| \leq 32 g$ is clearly finite.

\section{Classification of rings with toroidal unit graphs}

In this section, we determine, up to isomorphism, all finite commutative rings with nonzero identity whose unit graphs have genus one, that is, whose unit graphs are toroidal graphs.

Let $R$ be a finite commutative ring with nonzero identity such that $\gamma(G(R))=1$. Then, by Theorem 3.8, $R$ is isomorphic to either $S$ or $\mathbb{Z}_{2} \times S$, where $S$ is one of the finite rings mentioned in Table 2 . There are 36 such possibilities for $R$, among which we single out the ones whose unit graphs have genus 1 . For this purpose, the following result is very useful; in fact, in combination with Lemma 2.4, it helps

\begin{tabular}{|cc|}
\hline$|U(R)|$ & $S$ \\
\hline 7 & $\mathbb{F}_{8}$ \\
6 & $\mathbb{Z}_{7}, \mathbb{Z}_{9}, \mathbb{Z}_{3}[x] /\left\langle x^{2}\right\rangle, \mathbb{Z}_{3} \times \mathbb{F}_{4}, \mathbb{Z}_{4} \times \mathbb{F}_{4}, \mathbb{Z}_{2}[x] /\left\langle x^{2}\right\rangle \times \mathbb{F}_{4}$ \\
& $\mathbb{Z}_{5}($ for $t \neq 0), \mathbb{Z}_{8}, \mathbb{Z}_{2}[x] /\left\langle x^{3}\right\rangle, \mathbb{Z}_{2}[x] /\left\langle 2 x, x^{2}-2\right\rangle, \mathbb{Z}_{2}[x, y] /\langle x, y\rangle^{2}$, \\
4 & $\mathbb{Z}_{4}[x] /\langle 2, x\rangle^{2}, \mathbb{Z}_{3} \times \mathbb{Z}_{3}($ for $t \neq 0), \mathbb{Z}_{3} \times \mathbb{Z}_{4}, \mathbb{Z}_{3} \times \mathbb{Z}_{2}[x] /\left\langle x^{2}\right\rangle$, \\
& $\mathbb{Z}_{4} \times \mathbb{Z}_{4}, \mathbb{Z}_{4} \times \mathbb{Z}_{2}[x] /\left\langle x^{2}\right\rangle, \mathbb{Z}_{2}[x] /\left\langle x^{2}\right\rangle \times \mathbb{Z}_{2}[x] /\left\langle x^{2}\right\rangle$ \\
\hline
\end{tabular}

Table 2. Possible seed rings for toroidal unit graphs. 


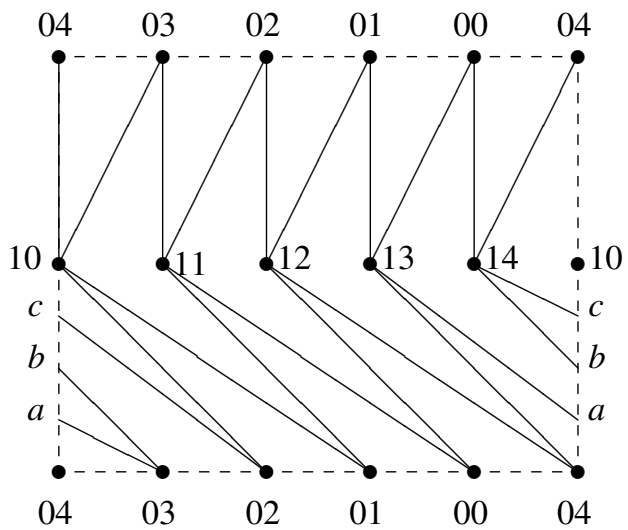

Figure 3. Embedding of the unit graph of $\mathbb{Z}_{2} \times \mathbb{Z}_{5}$ on a torus.

in determining some lower bounds for the genus of the unit graphs of rings of the type $\mathbb{Z}_{2} \times S$, where $S$ is a finite commutative ring with nonzero identity.

Lemma 4.1 [Ashrafi et al. 2010, Theorem 3.5]. Let $R$ be a finite commutative ring with nonzero identity, and $\mathfrak{m}$ be a maximal ideal of $R$ such that $R / \mathfrak{m} \cong \mathbb{Z}_{2}$. Then the unit graph $G(R)$ is a bipartite graph. Moreover, $G(R)$ is a complete bipartite graph if and only if $R$ is a local ring.

Let us now start the process of classification by looking at some toroidal unit graphs.

Proposition 4.2. $\gamma\left(G\left(\mathbb{Z}_{2} \times \mathbb{Z}_{5}\right)\right)=1$.

Proof. By Lemma 3.7, we have $\gamma\left(G\left(\mathbb{Z}_{2} \times \mathbb{Z}_{5}\right)\right) \geq 1$. But Figure 3 gives an embedding of the unit graph $G\left(\mathbb{Z}_{2} \times \mathbb{Z}_{5}\right)$ on a torus, and so $\gamma\left(G\left(\mathbb{Z}_{2} \times \mathbb{Z}_{5}\right)\right)=1$.

Proposition 4.3. $\gamma\left(G\left(\mathbb{Z}_{2} \times \mathbb{Z}_{3} \times \mathbb{Z}_{3}\right)\right)=1$.

Proof. By Lemma 3.7, we have $\gamma\left(G\left(\mathbb{Z}_{2} \times \mathbb{Z}_{3} \times \mathbb{Z}_{3}\right)\right) \geq 1$. But Figure 4 gives an embedding of $G\left(\mathbb{Z}_{2} \times \mathbb{Z}_{3} \times \mathbb{Z}_{3}\right)$ on a torus, and so $\gamma\left(G\left(\mathbb{Z}_{2} \times \mathbb{Z}_{3} \times \mathbb{Z}_{3}\right)\right)=1$.

Proposition 4.4. $\gamma\left(G\left(\mathbb{Z}_{3} \times \mathbb{Z}_{4}\right)\right)=\gamma\left(G\left(\mathbb{Z}_{3} \times \mathbb{Z}_{2}[x] /\left\langle x^{2}\right\rangle\right)\right)=1$.

Proof. By Lemma 3.7, we have $\gamma\left(G\left(\mathbb{Z}_{3} \times \mathbb{Z}_{4}\right)\right) \geq 1$. But Figure 5 gives an embedding of the unit graph $G\left(\mathbb{Z}_{3} \times \mathbb{Z}_{4}\right)$ on a torus, and so $\gamma\left(G\left(\mathbb{Z}_{3} \times \mathbb{Z}_{4}\right)\right)=1$. On the other hand, since the unit graph $G\left(\mathbb{Z}_{4}\right)$ is isomorphic to the unit graph $G\left(\mathbb{Z}_{2}[x] /\left\langle x^{2}\right\rangle\right)$, we have

$$
G\left(\mathbb{Z}_{3} \times \mathbb{Z}_{4}\right) \cong G\left(\mathbb{Z}_{3} \times \frac{\mathbb{Z}_{2}[x]}{\left\langle x^{2}\right\rangle}\right) .
$$

This completes the proof. 


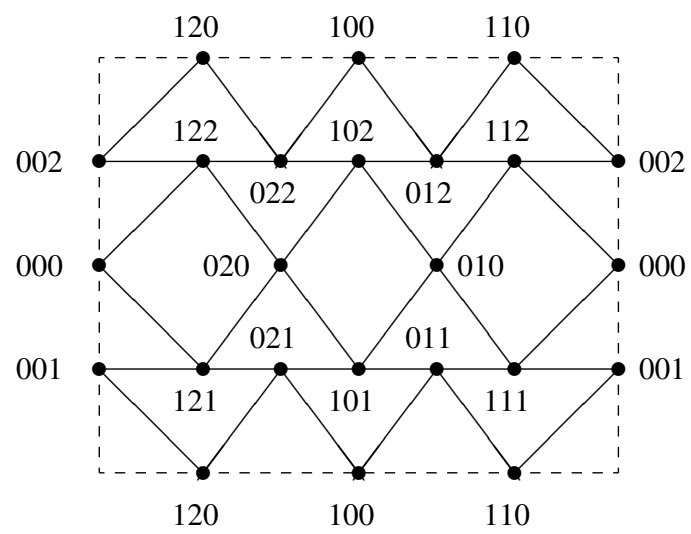

Figure 4. Embedding of the unit graph of $\mathbb{Z}_{2} \times \mathbb{Z}_{3} \times \mathbb{Z}_{3}$ on a torus.

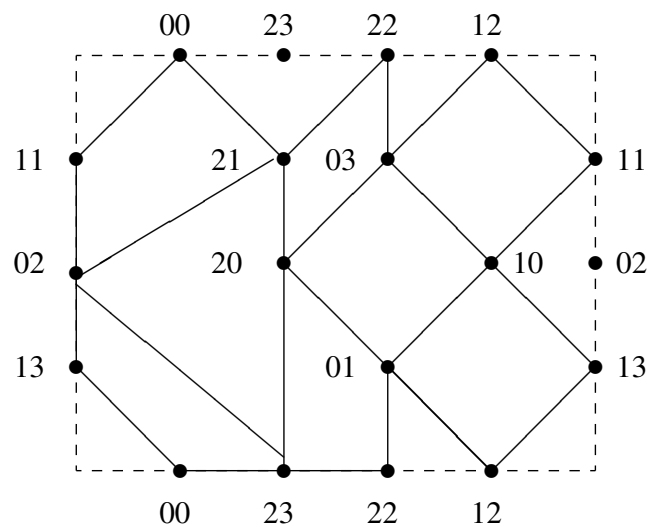

Figure 5. Embedding of the unit graph of $\mathbb{Z}_{3} \times \mathbb{Z}_{4}$ on a torus.

Proposition 4.5. If $S$ is one of the rings

$\mathbb{Z}_{7}, \quad \mathbb{Z}_{8}, \quad \frac{\mathbb{Z}_{2}[x]}{\left\langle x^{3}\right\rangle}, \quad \frac{\mathbb{Z}_{2}[x]}{\left\langle 2 x, x^{2}-2\right\rangle}, \quad \frac{\mathbb{Z}_{2}[x, y]}{\langle x, y\rangle^{2}}$ and $\frac{\mathbb{Z}_{4}[x]}{\langle 2, x\rangle^{2}}$,

then $\gamma(G(S))=1$.

Proof. Note that the unit graph $G\left(\mathbb{Z}_{7}\right)$ can be regarded as a subgraph of $K_{7}$, and so, by Lemmas 2.5 and 3.7, we have $\gamma\left(G\left(\mathbb{Z}_{7}\right)\right)=1$. On the other hand, each of the remaining rings is a local ring with 8 elements of which exactly 4 are zero-divisors, and so it follows from Lemma 4.1 that the associated unit graph of each of these rings is a complete bipartite graph, namely, $K_{4,4}$. The proof is now completed by Lemma 2.6.

Next we look at some unit graphs which have genus more than 1 .

Proposition 4.6. $\gamma\left(G\left(\mathbb{Z}_{2} \times \mathbb{Z}_{3} \times \mathbb{Z}_{4}\right)\right)=\gamma\left(G\left(\mathbb{Z}_{2} \times \mathbb{Z}_{3} \times \mathbb{Z}_{2}[x] /\left\langle x^{2}\right\rangle\right)\right)=2$. 
Proof. It is not difficult to see that $G\left(\mathbb{Z}_{2} \times \mathbb{Z}_{3} \times \mathbb{Z}_{4}\right)$ is isomorphic to the disjoint union of two copies of $G\left(\mathbb{Z}_{3} \times \mathbb{Z}_{4}\right)$. Therefore, by Lemma 2.2 and Proposition 4.4, we have $\gamma\left(G\left(\mathbb{Z}_{2} \times \mathbb{Z}_{3} \times \mathbb{Z}_{4}\right)\right)=2$. On the other hand, since the unit graph $G\left(\mathbb{Z}_{4}\right)$ is isomorphic to the unit graph $G\left(\mathbb{Z}_{2}[x] /\left\langle x^{2}\right\rangle\right)$, we have

$$
G\left(\mathbb{Z}_{2} \times \mathbb{Z}_{3} \times \mathbb{Z}_{4}\right) \cong G\left(\mathbb{Z}_{2} \times \mathbb{Z}_{3} \times \frac{\mathbb{Z}_{2}[x]}{\left\langle x^{2}\right\rangle}\right)
$$

This completes the proof.

Proposition 4.7. If $S$ is one of the rings

$$
\mathbb{Z}_{8}, \quad \frac{\mathbb{Z}_{2}[x]}{\left\langle x^{3}\right\rangle}, \quad \frac{\mathbb{Z}_{2}[x]}{\left\langle 2 x, x^{2}-2\right\rangle}, \quad \frac{\mathbb{Z}_{2}[x, y]}{\langle x, y\rangle^{2}} \text { and } \frac{\mathbb{Z}_{4}[x]}{\langle 2, x\rangle^{2}},
$$

then $\gamma\left(G\left(\mathbb{Z}_{2} \times S\right)\right)=2$. On the other hand, $\gamma\left(G\left(\mathbb{Z}_{2} \times \mathbb{Z}_{7}\right)\right) \geq 5$.

Proof. In view of the proof of Proposition 4.5, one may note that, for each of the given choices of $S$, the unit graph $G\left(\mathbb{Z}_{2} \times S\right)$ is isomorphic to the disjoint union of two copies of $K_{4,4}$. Hence, the first part follows from Lemmas 2.2 and 2.6.

For the second part, note that the unit graph $G\left(\mathbb{Z}_{2} \times \mathbb{Z}_{7}\right)$ is 6 -regular with 14 vertices. Also, by Lemma 4.1, it is bipartite, and so it has 42 edges. Therefore, by Lemma 2.4 , we have $\gamma\left(G\left(\mathbb{Z}_{2} \times \mathbb{Z}_{7}\right)\right) \geq 5$. This completes the proof.

Let us now recall that a subgraph $H$ of a graph $G$ is called a spanning subgraph if they have the same sets of vertices. A 1-regular spanning subgraph $H$ of a graph $G$ is called a perfect matching of $G$. Given a graph $G$ with a subgraph $H$, we write $G \backslash H$ to denote the subgraph of $G$ in which the vertex set is $V(G)$ and the edge set is $E(G) \backslash E(H)$.

Proposition 4.8. $\gamma\left(G\left(\mathbb{F}_{8}\right)\right)=2$ and $\gamma\left(G\left(\mathbb{Z}_{2} \times \mathbb{F}_{8}\right)\right) \geq 7$.

Proof. The unit graph $G\left(\mathbb{F}_{8}\right)$ is isomorphic to $K_{8}$, and so, by Lemma 2.5, we have $\gamma\left(G\left(\mathbb{F}_{8}\right)\right)=2$. On the other hand, the unit graph $G\left(\mathbb{Z}_{2} \times \mathbb{F}_{8}\right)$ has 16 vertices, and, by Lemma 4.1, it is bipartite. In fact, this graph is isomorphic to the graph $K_{8,8} \backslash M$, where $M$ is a perfect matching of $K_{8,8}$, and so it has 56 edges. Therefore, by Lemma 2.4, we have $\gamma\left(G\left(\mathbb{Z}_{2} \times \mathbb{F}_{8}\right)\right) \geq 7$.

Proposition 4.9. $\gamma\left(G\left(\mathbb{Z}_{3} \times \mathbb{F}_{4}\right)\right) \geq 2$ and $\gamma\left(G\left(\mathbb{Z}_{2} \times \mathbb{Z}_{3} \times \mathbb{F}_{4}\right)\right) \geq 7$.

Proof. Note that the unit graph $G:=G\left(\mathbb{Z}_{3} \times \mathbb{F}_{4}\right)$ is the union of two planar subgraphs plotted in Figure 6 which intersect at exactly four vertices, namely, $00,01,0 a$ and $0 a^{2}$ (indicated by bigger bullets). It is easy to see that the graph $G$ has 12 vertices and 36 edges. Moreover, it is a 6-regular graph. Also, it is easy to see that it is not a planar graph, as it contains a subdivision of $K_{5}$, namely, $\left\{20,21,2 a, 2 a^{2}, 0 a, 11,0 a^{2}, 20\right\}$. 

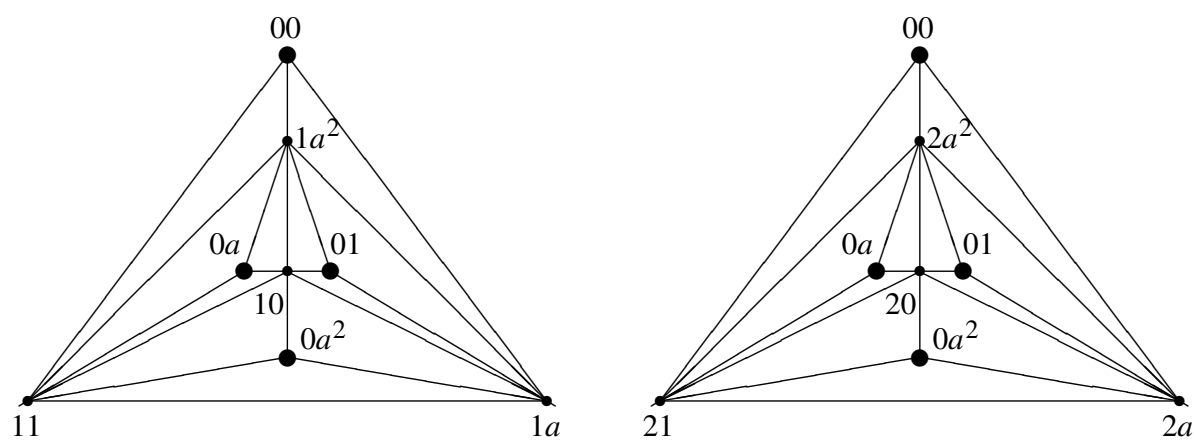

Figure 6. Two planar subgraphs of the unit graph of $\mathbb{Z}_{3} \times \mathbb{F}_{4}$.
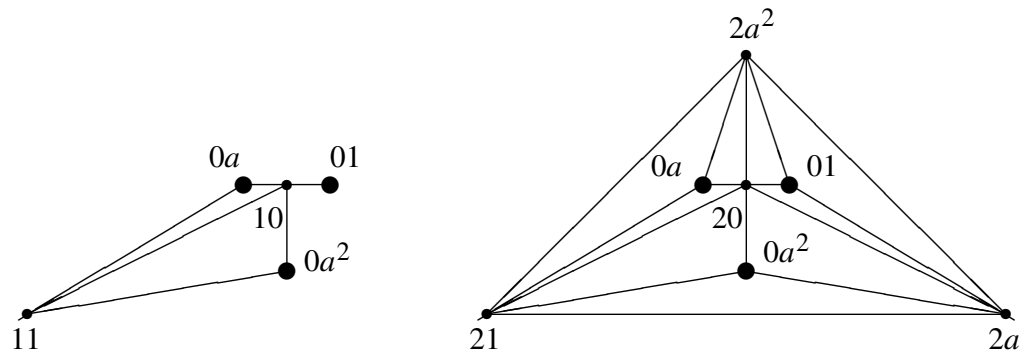

Figure 7. The graph $G\left(\mathbb{Z}_{3} \times \mathbb{F}_{4}\right)-\left\{00,1 a^{2}, 1 a, 00\right\}$.

Let us now assume that $G$ is toroidal, that is, $\gamma(G)=1$. Then, by Euler's formula, $G$ has $36-12=24$ faces. Also, note that $G$ is symmetrical in nature. We use Lemma 2.1 to show that every 3-cycle $C$ in $G$ is a face, and arrive at a contradiction.

First, let us consider a 3-cycle having empty interior. By symmetry, it is enough to take $C=\left\{00,1 a^{2}, 1 a, 00\right\}$. Then $G-C$ is given as indicated in Figure 7. Clearly, $G-C$ is nonplanar, as it contains a subdivision of $K_{5}$, namely, $\left\{20,21,2 a, 2 a^{2}\right.$, $\left.0 a, 11,0 a^{2}, 20\right\}$. Moreover, $C$ is chordless and $G-C$ is connected. Therefore, by Lemma 2.1, $C$ is a face. Note that there are 24 such 3-cycles in $G$.

Next, we consider the 3 -cycle $C=\left\{1 a^{2}, 10,1 a, 1 a^{2}\right\}$. Then $G-C$ is given as indicated in Figure 8. Again, it is clear that $G-C$ is nonplanar, as it contains a subdivision of $K_{5}$, namely, $\left\{20,21,2 a, 2 a^{2}, 0 a, 11,0 a^{2}, 20\right\}$. Moreover, $C$ is chordless and $G-C$ is connected. Therefore, by Lemma 2.1, $C$ is a face.

Since we have already found 25 faces, our assumption that $G$ is toroidal is wrong. Hence, we conclude that $\gamma\left(G\left(\mathbb{Z}_{3} \times \mathbb{F}_{4}\right)\right) \geq 2$.

For the second part, note that the unit graph $G\left(\mathbb{Z}_{2} \times \mathbb{Z}_{3} \times \mathbb{F}_{4}\right)$ is 6-regular with 24 vertices. Also, by Lemma 4.1, it is bipartite, and so it has 72 edges. Therefore, by Lemma 2.4 , we have $\gamma\left(G\left(\mathbb{Z}_{2} \times \mathbb{Z}_{3} \times \mathbb{F}_{4}\right)\right) \geq 7$. This completes the proof.

Arguing in the same manner as above, we also have the following result. 


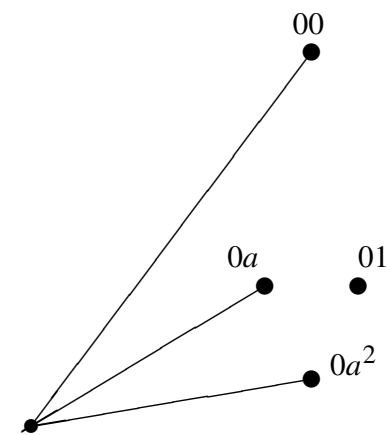

11

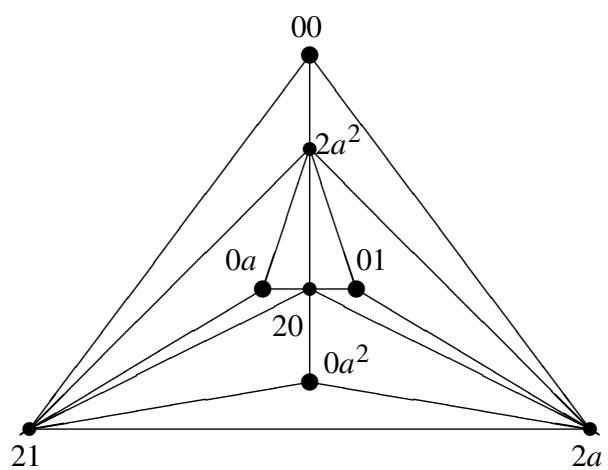

21

Figure 8. The graph $G\left(\mathbb{Z}_{3} \times \mathbb{F}_{4}\right)-\left\{1 a^{2}, 10,1 a, 1 a^{2}\right\}$.

Proposition 4.10. If $S$ is one of the rings $\mathbb{Z}_{9}$ and $\mathbb{Z}_{3}[x] /\left\langle x^{2}\right\rangle$, then $\gamma(G(S)) \geq 2$ and $\gamma\left(G\left(\mathbb{Z}_{2} \times S\right)\right) \geq 6$.

Proof. Note that the unit graphs of the rings $\mathbb{Z}_{9}$ and $\mathbb{Z}_{3}[x] /\left\langle x^{2}\right\rangle$ are isomorphic. Therefore, it is enough to prove the result only for $\mathbb{Z}_{9}$. It is easy to see, from Figure 9, that the unit graph $G\left(\mathbb{Z}_{9}\right)$ is a nonplanar graph with 9 vertices and 24 edges. Moreover, the number of 3-cycles in it is 20 . If $C$ is one such 3-cycle, then it is easy to see that $C$ is chordless, and $G\left(\mathbb{Z}_{9}\right)-C$ is connected and nonplanar. In fact, $G\left(\mathbb{Z}_{9}\right)-C$ is either a subdivision of $K_{5}$ or has a subgraph isomorphic to $K_{3,3}$, depending on whether the 3-cycle $C$ contains one or none of the vertices 0,3 and 6. Therefore, if $G\left(\mathbb{Z}_{9}\right)$ is toroidal, then it follows from Lemma 2.1 that every 3 -cycle in $G\left(\mathbb{Z}_{9}\right)$ is a face, and so $G\left(\mathbb{Z}_{9}\right)$ has at least 20 faces, whereas from Euler's formula it follows that $G\left(\mathbb{Z}_{9}\right)$ has 15 faces. Hence, we have $\gamma\left(G\left(\mathbb{Z}_{9}\right)\right) \geq 2$.

For the second part, note that the unit graph $G\left(\mathbb{Z}_{2} \times \mathbb{Z}_{9}\right)$ is 6-regular with 18 vertices. Also, by Lemma 4.1, it is bipartite, and so it has 54 edges. Therefore, by Lemma 2.4 , we have $\gamma\left(G\left(\mathbb{Z}_{2} \times \mathbb{Z}_{9}\right)\right) \geq 6$. This completes the proof.

Proposition 4.11. If $S$ is one of the rings

$$
\mathbb{Z}_{4} \times \mathbb{Z}_{4}, \quad \mathbb{Z}_{4} \times \frac{\mathbb{Z}_{2}[x]}{\left\langle x^{2}\right\rangle} \text { and } \frac{\mathbb{Z}_{2}[x]}{\left\langle x^{2}\right\rangle} \times \frac{\mathbb{Z}_{2}[x]}{\left\langle x^{2}\right\rangle},
$$

then $\gamma(G(S))=2$ and $\gamma\left(G\left(\mathbb{Z}_{2} \times S\right)\right)=4$.

Proof. Consider the following two subsets of the vertex set of $G\left(\mathbb{Z}_{4} \times \mathbb{Z}_{4}\right)$ :

$$
\begin{aligned}
& V_{1}=\{(0,0),(0,2),(2,0),(2,2),(1,1),(1,3),(3,1),(3,3)\}, \\
& V_{2}=\{(0,1),(0,3),(2,1),(2,3),(1,0),(1,2),(3,0),(3,2)\} .
\end{aligned}
$$

It is easy to see that the two subgraphs $\left\langle V_{1}\right\rangle$ and $\left\langle V_{2}\right\rangle$ are disjoint, and their union is $G\left(\mathbb{Z}_{4} \times \mathbb{Z}_{4}\right)$. Moreover, $\left\langle V_{1}\right\rangle \cong\left\langle V_{2}\right\rangle \cong K_{4,4}$. Therefore, it follows from Lemma 2.2 and Lemma 2.6 that $\gamma\left(G\left(\mathbb{Z}_{4} \times \mathbb{Z}_{4}\right)\right)=2$. Also, it is easy to see that $G\left(\mathbb{Z}_{2} \times \mathbb{Z}_{4} \times \mathbb{Z}_{4}\right)$ 


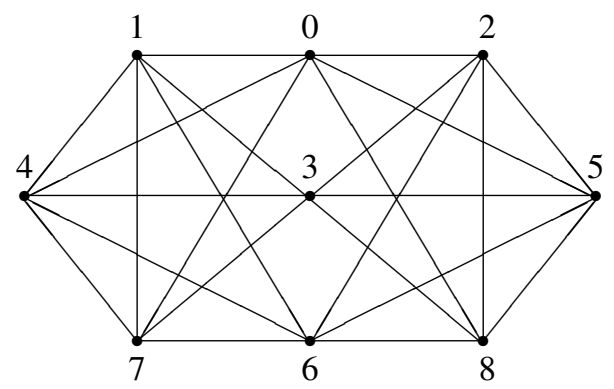

Figure 9. The unit graph of $\mathbb{Z}_{9}$.

is isomorphic to the disjoint union of two copies of $G\left(\mathbb{Z}_{4} \times \mathbb{Z}_{4}\right)$. Therefore, in view of Lemma 2.2, it follows that $\gamma\left(G\left(\mathbb{Z}_{2} \times \mathbb{Z}_{4} \times \mathbb{Z}_{4}\right)\right)=4$. On the other hand, since the unit graph $G\left(\mathbb{Z}_{4}\right)$ is isomorphic to the unit graph $G\left(\mathbb{Z}_{2}[x] /\left\langle x^{2}\right\rangle\right)$, we have

$$
G\left(\mathbb{Z}_{4} \times \mathbb{Z}_{4}\right) \cong G\left(\mathbb{Z}_{4} \times \frac{\mathbb{Z}_{2}[x]}{\left\langle x^{2}\right\rangle}\right) \cong G\left(\frac{\mathbb{Z}_{2}[x]}{\left\langle x^{2}\right\rangle} \times \frac{\mathbb{Z}_{2}[x]}{\left\langle x^{2}\right\rangle}\right)
$$

and

$$
G\left(\mathbb{Z}_{2} \times \mathbb{Z}_{4} \times \mathbb{Z}_{4}\right) \cong G\left(\mathbb{Z}_{2} \times \mathbb{Z}_{4} \times \frac{\mathbb{Z}_{2}[x]}{\left\langle x^{2}\right\rangle}\right) \cong G\left(\mathbb{Z}_{2} \times \frac{\mathbb{Z}_{2}[x]}{\left\langle x^{2}\right\rangle} \times \frac{\mathbb{Z}_{2}[x]}{\left\langle x^{2}\right\rangle}\right) .
$$

Hence, the result follows.

Proposition 4.12. If $S$ is one of the rings $\mathbb{Z}_{4} \times \mathbb{F}_{4}$ and $\mathbb{Z}_{2}[x] /\left\langle x^{2}\right\rangle \times \mathbb{F}_{4}$, then $\gamma(G(S)) \geq 2$ and $\gamma\left(G\left(\mathbb{Z}_{2} \times S\right)\right) \geq 9$.

Proof. Note that $G\left(\mathbb{Z}_{4}\right)$ is a spanning subgraph of $G\left(\mathbb{F}_{4}\right)$. This implies that $G\left(\mathbb{Z}_{4} \times \mathbb{Z}_{4}\right)$ is an spanning subgraph of $G\left(\mathbb{Z}_{4} \times \mathbb{F}_{4}\right)$. Therefore, by Proposition 4.11, we have $\gamma\left(G\left(\mathbb{Z}_{4} \times \mathbb{F}_{4}\right)\right) \geq 2$. Also note that the unit graph $G\left(\mathbb{Z}_{2} \times \mathbb{Z}_{4} \times \mathbb{F}_{4}\right)$ is 6-regular with 32 vertices. Moreover, by Lemma 4.1, it is bipartite, and so it has 96 edges. Therefore, by Lemma 2.4, we have $\gamma\left(G\left(\mathbb{Z}_{2} \times \mathbb{Z}_{4} \times \mathbb{F}_{4}\right)\right) \geq 9$. On the other hand, since the unit graph $G\left(\mathbb{Z}_{4}\right)$ is isomorphic to the unit graph $G\left(\mathbb{Z}_{2}[x] /\left\langle x^{2}\right\rangle\right)$, we have

$$
G\left(\mathbb{Z}_{4} \times \mathbb{F}_{4}\right) \cong G\left(\frac{\mathbb{Z}_{2}[x]}{\left\langle x^{2}\right\rangle} \times \mathbb{F}_{4}\right)
$$

and

$$
G\left(\mathbb{Z}_{2} \times \mathbb{Z}_{4} \times \mathbb{F}_{4}\right) \cong G\left(\mathbb{Z}_{2} \times \frac{\mathbb{Z}_{2}[x]}{\left\langle x^{2}\right\rangle} \times \mathbb{F}_{4}\right) .
$$

This completes the proof.

Let us now summarize what we have achieved so far: If $S$ is a finite commutative ring with nonzero identity, then, with $\gamma_{S}=\gamma(G(S))$ and $\gamma_{2 S}=\gamma\left(G\left(\mathbb{Z}_{2} \times S\right)\right)$, one has Table 3. 


\begin{tabular}{ccc|}
\hline$S$ & $\gamma_{S}$ & $\gamma_{2 S}$ \\
\hline $\mathbb{Z}_{5}, \mathbb{Z}_{3} \times \mathbb{Z}_{3}$ & 0 & 1 \\
$\mathbb{Z}_{3} \times \mathbb{Z}_{4}, \mathbb{Z}_{3} \times \mathbb{Z}_{2}[x] /\left\langle x^{2}\right\rangle, \mathbb{Z}_{8}, \mathbb{Z}_{2}[x] /\left\langle x^{3}\right\rangle$, & 1 & 2 \\
$\mathbb{Z}_{2}[x] /\left\langle 2 x, x^{2}-2\right\rangle, \mathbb{Z}_{2}[x, y] /\langle x, y\rangle^{2}, \mathbb{Z}_{4}[x] /\langle 2, x\rangle^{2}$ & & \\
$\mathbb{Z}_{7}$ & 1 & $\geq 5$ \\
$\mathbb{F}_{8}$ & 2 & $\geq 7$ \\
$\mathbb{Z}_{3} \times \mathbb{F}_{4}$ & $\geq 2$ & $\geq 7$ \\
$\mathbb{Z}_{9}, \mathbb{Z}_{3}[x] /\left\langle x^{2}\right\rangle$ & $\geq 2$ & $\geq 6$ \\
$\mathbb{Z}_{4} \times \mathbb{Z}_{4}, \mathbb{Z}_{4} \times \mathbb{Z}_{2}[x] /\left\langle x^{2}\right\rangle, \mathbb{Z}_{2}[x] /\left\langle x^{2}\right\rangle \times \mathbb{Z}_{2}[x] /\left\langle x^{2}\right\rangle$ & 2 & 4 \\
$\mathbb{Z}_{4} \times \mathbb{F}_{4}, \mathbb{Z}_{2}[x] /\left\langle x^{2}\right\rangle \times \mathbb{F}_{4}$ & $\geq 2$ & $\geq 9$ \\
\hline
\end{tabular}

Table 3. Genus of some unit graphs.

Finally, using Table 3 and Theorem 3.8, one easily obtains the following classification theorem.

Theorem 4.13. Let $R$ be a finite commutative ring with nonzero identity. Then the unit graph $G(R)$ is a toroidal graph if and only if $R$ is isomorphic to one of

$$
\begin{array}{ccccc}
\mathbb{Z}_{2} \times \mathbb{Z}_{5}, & \mathbb{Z}_{2} \times \mathbb{Z}_{3} \times \mathbb{Z}_{3}, & \mathbb{Z}_{3} \times \mathbb{Z}_{4}, & \mathbb{Z}_{3} \times \frac{\mathbb{Z}_{2}[x]}{\left\langle x^{2}\right\rangle}, \quad \mathbb{Z}_{8}, \\
\frac{\mathbb{Z}_{2}[x]}{\left\langle x^{3}\right\rangle}, & \frac{\mathbb{Z}_{2}[x]}{\left\langle 2 x, x^{2}-2\right\rangle}, & \frac{\mathbb{Z}_{2}[x, y]}{\langle x, y\rangle^{2}}, & \frac{\mathbb{Z}_{4}[x]}{\langle 2, x\rangle^{2}} \text { or } \quad \mathbb{Z}_{7} .
\end{array}
$$

\section{Acknowledgment}

The authors are grateful to the anonymous referee for bringing to their notice an inadvertent omission in Theorem 3.8, for making many constructive suggestions, and for supplying some additional references.

\section{References}

[Anderson and Livingston 1999] D. F. Anderson and P. S. Livingston, "The zero-divisor graph of a commutative ring", J. Algebra 217:2 (1999), 434-447. MR 2000e:13007 Zbl 0941.05062

[Anderson and Naseer 1993] D. D. Anderson and M. Naseer, "Beck's coloring of a commutative ring”, J. Algebra 159:2 (1993), 500-514. MR 94e:13009 Zbl 0798.05067

[Anderson et al. 2011] D. F. Anderson, M. C. Axtell, and J. A. Stickles, "Zero-divisor graphs in commutative rings", pp. 23-45 in Commutative algebra: Noetherian and non-Noetherian perspectives, edited by M. Fontana et al., Springer, New York, 2011. MR 2012b:13023 Zbl 1225.13002

[Archdeacon 1996] D. Archdeacon, "Topological graph theory: a survey", Congr. Numer. 115 (1996), 5-54. MR 98g:05044 Zbl 0897.05026 
[Ashrafi et al. 2010] N. Ashrafi, H. R. Maimani, M. R. Pournaki, and S. Yassemi, "Unit graphs associated with rings", Comm. Algebra 38:8 (2010), 2851-2871. MR 2011j:13010 Zbl 1219.05150

[Battle et al. 1962] J. Battle, F. Harary, Y. Kodama, and J. W. T. Youngs, "Additivity of the genus of a graph”, Bull. Amer. Math. Soc. 68 (1962), 565-568. MR 27 \#5247 Zbl 0142.41501

[Beck 1988] I. Beck, "Coloring of commutative rings", J. Algebra 116:1 (1988), 208-226. MR 89i: 13006 Zbl 0654.13001

[Chartrand and Oellermann 1993] G. Chartrand and O. R. Oellermann, Applied and algorithmic graph theory, McGraw-Hill, New York, 1993. MR 1211413

[Corbas and Williams 2000a] B. Corbas and G. D. Williams, "Rings of order $p^{5}$, I: Nonlocal rings", J. Algebra 231:2 (2000), 677-690. MR 2001h:16020a Zbl 1017.16014

[Corbas and Williams 2000b] B. Corbas and G. D. Williams, "Rings of order $p^{5}$, II: Local rings", $J$. Algebra 231:2 (2000), 691-704. MR 2001h:16020b Zbl 1017.16015

[Grimaldi 1990] R. P. Grimaldi, "Graphs from rings", Congr. Numer. 71 (1990), 95-103. MR 90m: 05122 Zbl 0747.05091

[Maimani et al. 2010a] H. R. Maimani, M. R. Pournaki, and S. Yassemi, "A class of weakly perfect graphs", Czechoslovak Math. J. 60(135):4 (2010), 1037-1041. MR 2011h:05104 Zbl 1224.05376

[Maimani et al. 2010b] H. R. Maimani, M. R. Pournaki, and S. Yassemi, "Rings which are generated by their units: a graph theoretical approach", Elem. Math. 65:1 (2010), 17-25. MR 2011d:13039 Zbl 1209.13031

[Maimani et al. 2010c] H. R. Maimani, M. R. Pournaki, and S. Yassemi, "Weakly perfect graphs arising from rings", Glasg. Math. J. 52:3 (2010), 417-425. MR 2011h:05099 Zbl 1243.05085

[Maimani et al. 2011a] H. R. Maimani, M. R. Pournaki, A. Tehranian, and S. Yassemi, "Graphs attached to rings revisited", Arab. J. Sci. Eng. 36:6 (2011), 997-1011. MR 2845527

[Maimani et al. 2011b] H. R. Maimani, M. R. Pournaki, and S. Yassemi, "Necessary and sufficient conditions for unit graphs to be Hamiltonian”, Pacific J. Math. 249:2 (2011), 419-429. MR 2012b:05164 Zbl 1214.05074

[Maimani et al. 2012] H. R. Maimani, C. Wickham, and S. Yassemi, "Rings whose total graphs have genus at most one”, Rocky Mountain J. Math. 42:5 (2012), 1551-1560. MR 3001816 Zbl 1254.05164

[McDonald 1974] B. R. McDonald, Finite rings with identity, Pure and Applied Mathematics 28, Marcel Dekker, New York, 1974. MR 50 \#7245 Zbl 0294.16012

[Neufeld and Myrvold 1997] E. Neufeld and W. Myrvold, "Practical toroidality testing", pp. 574-580 in Proceedings of the Eighth Annual ACM-SIAM Symposium on Discrete Algorithms (New Orleans, LA, 1997), edited by M. Saks, ACM, New York, 1997. MR 1447705

[Raghavendran 1969] R. Raghavendran, "Finite associative rings", Compositio Math. 21:2 (1969), 195-229. MR 40 \#174 Zbl 0179.33602

[White 1973] A. T. White, Graphs, groups and surfaces, North-Holland Mathematics Studies 8, North-Holland, Amsterdam, 1973. MR 49 \#4783 Zbl 0268.05102

[Wickham 2008] C. Wickham, "Classification of rings with genus one zero-divisor graphs", Comm. Algebra 36:2 (2008), 325-345. MR 2009f:13010 Zbl 1137.13015 
A. K. DAS

DEPARTMENT OF MATHEMATICS

NORTH-EASTERN HILl UNIVERSITY

PERMANENT CAMPUS

SHILLONG-793022

MEGHALAYA

INDIA

akdasnehu@gmail.com

H. R. MAIMANI

Mathematics Section

DEPARTMENT OF BASIC SCIENCES

Shahid RAJAEE TEACHER TRAINING UNIVERSITY

P.O. BOX 16785-163

and

SCHOOL OF MATHEMATICS

INSTITUTE FOR RESEARCH IN FUNDAMENTAL SCIENCES (IPM)

P.O. BOX 19395-5746

TEHRAN

IRAN

maimani@ipm.ir

M. R. POURNAKI

DEPARTMENT OF MATHEMATICAL SCIENCES

SHARIF UNIVERSITY OF TECHNOLOGY

P.O. BOX 11155-9415

and

SCHOOL OF MATHEMATICS

INSTITUTE FOR RESEARCH IN FUNDAMENTAL SCIENCES (IPM)

P.O. BOX 19395-5746

TEHRAN

IRAN

pournaki@ipm.ir

S. YASSEMI

School of Mathematics, Statistics and Computer Science

COLLEGE OF SCIENCE

UNIVERSITY OF TEHRAN

and

SCHOOL OF MATHEMATICS

INSTITUTE FOR RESEARCH IN FUNDAMENTAL SCIENCES (IPM)

P.O. BOX 19395-5746

TEHRAN

IRAN

yassemi@ipm.ir 


\title{
PACIFIC JOURNAL OF MATHEMATICS
}

\author{
msp.org/pjm
}

Founded in 1951 by E. F. Beckenbach (1906-1982) and F. Wolf (1904-1989)

\section{EDITORS}

Don Blasius (Managing Editor)

Department of Mathematics

University of California

Los Angeles, CA 90095-1555

blasius@math.ucla.edu

\author{
Paul Balmer \\ Department of Mathematics \\ University of California \\ Los Angeles, CA 90095-1555 \\ balmer@math.ucla.edu \\ Robert Finn \\ Department of Mathematics \\ Stanford University \\ Stanford, CA 94305-2125 \\ finn@math.stanford.edu \\ Sorin Popa \\ Department of Mathematics \\ University of California \\ Los Angeles, CA 90095-1555 \\ popa@math.ucla.edu
}

\author{
Vyjayanthi Chari \\ Department of Mathematics \\ University of California \\ Riverside, CA 92521-0135 \\ chari@math.ucr.edu \\ Kefeng Liu \\ Department of Mathematics \\ University of California \\ Los Angeles, CA 90095-1555 \\ liu@math.ucla.edu \\ Jie Qing \\ Department of Mathematics \\ University of California \\ Santa Cruz, CA 95064 \\ qing@ cats.ucsc.edu
}

\section{PRODUCTION}

Silvio Levy, Scientific Editor, production@msp.org

\section{SUPPORTING INSTITUTIONS}

ACADEMIA SINICA, TAIPEI

CALIFORNIA INST. OF TECHNOLOGY

INST. DE MATEMÁTICA PURA E APLICADA

KEIO UNIVERSITY

MATH. SCIENCES RESEARCH INSTITUTE

NEW MEXICO STATE UNIV.

OREGON STATE UNIV.

\author{
STANFORD UNIVERSITY \\ UNIV. OF BRITISH COLUMBIA \\ UNIV. OF CALIFORNIA, BERKELEY \\ UNIV. OF CALIFORNIA, DAVIS \\ UNIV. OF CALIFORNIA, LOS ANGELES \\ UNIV. OF CALIFORNIA, RIVERSIDE \\ UNIV. OF CALIFORNIA, SAN DIEGO \\ UNIV. OF CALIF., SANTA BARBARA
}

\author{
Daryl Cooper \\ Department of Mathematics \\ University of California \\ Santa Barbara, CA 93106-3080 \\ cooper@math.ucsb.edu \\ Jiang-Hua Lu \\ Department of Mathematics \\ The University of Hong Kong \\ Pokfulam Rd., Hong Kong \\ jhlu@maths.hku.hk \\ Paul Yang \\ Department of Mathematics \\ Princeton University \\ Princeton NJ 08544-1000 \\ yang@math.princeton.edu
}

These supporting institutions contribute to the cost of publication of this Journal, but they are not owners or publishers and have no responsibility for its contents or policies.

See inside back cover or msp.org/pjm for submission instructions.

The subscription price for 2014 is US $\$ 410 /$ year for the electronic version, and \$535/year for print and electronic.

Subscriptions, requests for back issues and changes of subscribers address should be sent to Pacific Journal of Mathematics, P.O. Box 4163, Berkeley, CA 94704-0163, U.S.A. The Pacific Journal of Mathematics is indexed by Mathematical Reviews, Zentralblatt MATH, PASCAL CNRS Index, Referativnyi Zhurnal, Current Mathematical Publications and Web of Knowledge (Science Citation Index).

The Pacific Journal of Mathematics (ISSN 0030-8730) at the University of California, c/o Department of Mathematics, 798 Evans Hall \#3840, Berkeley, CA 94720-3840, is published twelve times a year. Periodical rate postage paid at Berkeley, CA 94704, and additional mailing offices. POSTMASTER: send address changes to Pacific Journal of Mathematics, P.O. Box 4163, Berkeley, CA 94704-0163.

PJM peer review and production are managed by EditFLOW ${ }^{\circledR}$ from Mathematical Sciences Publishers.

\section{PUBLISHED BY}

\section{mathematical sciences publishers \\ nonprofit scientific publishing}

http://msp.org/

(C) 2014 Mathematical Sciences Publishers 


\title{
PACIFIC JOURNAL OF MATHEMATICS
}

\author{
Volume $268 \quad$ No. $2 \quad$ April 2014
}

In memoriam: Jonathan Rogawski

DON BLASIUS, DINAKAR RAMAKRISHNAN and V. S. VARADARAJAN

Formes modulaires sur la $\mathbb{Z}_{p}$-extension cyclotomique de $\mathbb{Q}$

LAURENT CLOZEL

Weight zero Eisenstein cohomology of Shimura varieties via Berkovich

275 spaces

MICHAEL HARRIS

$\Lambda$-adic Barsotti-Tate groups

HARUZO HIDA

Le flot géodésique des quotients géométriquement finis des géométries de

Hilbert

MickaËl CRAMPON and LUdOViC MARQuis

Nonplanarity of unit graphs and classification of the toroidal ones

A. K. Das, H. R. Maimani, M. R. Pournaki and S. Yassemi

Discrete semiclassical orthogonal polynomials of class one

DIEGO DOMINICI and FRANCISCO MARCELLÁN

A note on conformal Ricci flow

PENG LU, Jie QING and Yu Zheng

On representations of $\mathrm{GL}_{2 n}(F)$ with a symplectic period

ARNAB Mitra

Linked triples of quaternion algebras

ALEXANDER S. SIVATSKI

Finite nonsolvable groups with many distinct character degrees

Hung P. TONG-VIET

Errata to "Dynamics of asymptotically hyperbolic manifolds"

JULIE ROWLETT

Erratum to "Singularities of the projective dual variety" 